\title{
Detection of carbapenemase-producing Enterobacteriaceae in rectal surveillance cultures in non-hospitalized patients
}

\author{
Amal Mohamed Sayed*, Iman Kamal Behiry, Rasha Hamed Elsherief and Sara Ali Elsir
}

\begin{abstract}
Background: Carbapenem-resistant Enterobacteriaceae have been increasingly reported worldwide since their first identification more than 20 years ago. The early identification of carriers and implementing of cohorting strategies is the only means to prevent nosocomial outbreaks caused by carbapenemase.The aim of this work is to evaluate the prevalence of intestinal colonization with carbapenemase-producing Enterobacteriaceae (CPE) in non-hospitalized patients and assesses a microbiological protocol for screening these isolates in fecal material.

Methods: This study was conducted on patients seen at Kasr Alaini hospital and investigated at microbiology laboratory in Cairo University Hospitals, from May 2013 to October 2013. The study was conducted on 600 patients; 450 of them were non hospitalized patients (group I) and came for regular checkup, and 150 were inpatients as control group (group II) from different hospital departments.

Results: Based on questionnaire feedback, history and clinical findings, certain patients were suspected to have CPE. Group I includes 337 (56.2\%) males and 113 (18.8\%) females and group II includes 83 (13.8\%) males and 67 (11.2\%) females. Age of group I ranged from 18 to 82 years with a mean of 44 years, while the age of group II ranged from 10 to 77 years with a mean of 37 years. Out of 600 fecal samples (450 from group I and 150 from group II) 12 (carriage rate 2\%) were positive by disk diffusion methods, Chrom agar-KPC and or Modified Hodge Test (MHT) and PCR. Two of them were non hospitalized patients (group I) (carriage rate $0.33 \%$ ) and 10 were hospitalized patients (control group) (carriage rate 1. 7\%) from different hospital departments.
\end{abstract}

Conclusions: We describe a fecal carriage of carbapenam producing Enterobactericae ,all of them are NAD producers orOXA-48 producers in patients non infected by these organisms.

Keywords: Enterobacteriaceae, Klebsiella pneumoniae carbapenemase (KPC), Metallo-beta-lactamase, New Delhi metallobeta-lactamase-1, Oxacillinase OXA-48

\section{Background}

$\mathrm{CPE}$, which stands for carbapenemase-producing-Enterobacteriaceae, are a family of germs that are difficult to treat because they have high levels of resistance to antibiotics. Klebsiella species and Escherichia coli (E. coli) are examples of Enterobacteriaceae, a normal part of the human gut bacteria, that can become carbapenem-resistant. Types of CPE are sometimes known as KPC (Klebsiella pneumoniae carbapenemase) and NDM (New Delhi metallo-betalactamase). KPC and NDM are enzymes that break down carbapenems and make them ineffective. Both of these

\footnotetext{
* Correspondence: imaging70@gmail.com

Clinical and Chemical Pathology Department, Faculty of Medicine, Cairo University, Cairo, Egypt
}

enzymes, as well as the enzyme VIM (Verona integronmediated metallo- $\beta$-lactamase) have also been reported in pseudomonas (CDC 2015). Carbapenemases are $\beta$ lactamases with versatile hydrolytic capacities. They have the ability to hydrolyze penicillins, cephalosporins, monobactams, and carbapenems. Bacteria producing these $\beta$-lactamases may cause serious infections in which the carbapenemase activity renders many ß-lactams ineffective (Queenan and Bush 2007a). Carbapenemaseproducing Enterobacteriaceae (CPE) isolates have been increasingly reported in Europe (Grundmann and et al. 2010). They include enzymes belonging to the Ambler classes A (KPC types), B (VIM, IMP, and more recently NDM enzymes), and D (OXA-48). With the exception of OXA-48, these enzymes confer high-level resistance to most 
$\beta$-lactam compounds, such as penicillins and cephalosporins, but variably affect susceptibility to carbapenems (Tato et al. 2010). To date, three main classes of carbapenemases have been identified (Queenan and Bush 2007a). Ambler class A beta-lactamases are enzymes that can be either chromosomally encoded or plasmid encoded Verona integronencoded metallo- $\beta$-lactamase (blaVIM), and the New Delhi metallo- $\beta$-lactamase (blaNDM) belong to class B metallo- $\beta$ lactamases (MBLs). The characterization of blaNDM was firstly published in December 2009 (Yong et al. 2009) and its presence was then detected in almost the entire world (Cornaglia et al. 2011). BlaOXA-48 carbapenemases belong to Ambler class D. The prevalence of CPE in Spain remains low and is mainly related to VIM-producing isolates (Sánchez-Romero et al. 2012). Nevertheless, KPC-2-, KPC-3-, NDM-1-, and OXA-48-producing Enterobacteriaceae were recently detected in Spain (Solé et al. 2011). Fecal carriage of CPE isolates has been investigated rarely compared with carriage of isolates producing extended-spectrum $\beta$-lactamases (ESBLs) (Tórtola et al. 2005), particularly among patients not selected for their relation to outbreak cases. In addition, few surveillance culture recommendations are available for detecting CPE (Ruppé et al. 2011).

\section{Methods}

This study was conducted on patients seen at Kasr Alaini hospital and investigated at microbiology laboratory in Cairo University Hospitals, from May 2013 to October 2013.

\section{Patients}

The study was conducted on 600 patients; 450 of them were non hospitalized patients (group I) and came for regular checkup, full history was taken from them with the help of specifically structured questionnaire that addressed certain areas of interest regarding the age, chronic medical illness and previous history of hospitalization within the last two months before study and if there was any operative history together with antibiotic usage. Patients with any GIT symptoms especially diarrhea were excluded from the study. Stool samples were also collected from 150 inpatients as control group (group II) from different wards at the hospital, all of them were not complaining from any GIT symptoms. Based on questionnaire feedback and clinical findings, certain patients were suspected to have CPE.

\section{Methods}

A. Specimen: stool specimens obtained from both groups (group I and group II) were cultured directly on routine media as MacConkey (Oxoid Co. England) and blood agar (OxoidCo.England). Isolated gram negative bacilli confirmed by gram stain and biochemical reaction. Oxidase test was done for isolates proved to be non fermentor.
B. Screening isolates for CRE:

1. Disk diffusion: this was detected by using imipenem, meropenem disk diffusion method. Results were categorized as sensitive, intermediate and resistant as Clinical Laboratory Standard Institute (CLSI) guidelines 2012, (imipenem $10 \mu \mathrm{g}: \mathrm{S}: \geq 23$, I: $20-22$, R: $\leq 19$ ), (meropenem: $10 \mu \mathrm{g}: \mathrm{S}: \geq 23$, I: $20-22, \mathrm{R}: \leq 19)$.

2. Chromagar KPC: The Chromagar KPC detects enterobacteriacea that express or produce enzymes like KPC, OXA-48, and NDM-1. Chromagar-KPC (Chromagar of Dr.A.Rambach) was prepared by using Chromagar orientation powder base and Chromagar KPC supplement:

A-Preparation of Chrom agar was done by suspending the medium in the proportion of $33.0 \mathrm{~g} / \mathrm{L}$ of purified water, then powder was dispersed slowly in water by rotating for swelling of the agar, the mixture was heated and brought to boiling $\left(100{ }^{\circ} \mathrm{C}\right)$ while swirling or stirring regularly and autoclaved at $121{ }^{\circ} \mathrm{C}$ for $15 \mathrm{~min}$, and the mixture was cooled in a water bath to $45-50{ }^{\circ} \mathrm{C}$ with gentle swirling.

$\mathrm{B}$ - The required supplement was aliquoted for $400 \mathrm{mg} / \mathrm{l}$ final, then purified water was added to make a supplement solution at $40 \mathrm{mg} / \mathrm{ml}$; the appearing suspension was opaqueyellowish. The supplement was vortexed to homogenize and added, in the proportion $10 \mathrm{ml} / \mathrm{l}$ of final media, to melted Chromagar orientation cooled at $45 / 50{ }^{\circ} \mathrm{C}$ and stirred to make Chromagar KPC that was finally poured into Petri dishes, cooled and stored in the refrigeration $\left(2 / 8{ }^{\circ} \mathrm{C}\right)$ with protection from light and dehydration. Life spans for prepared plates were two weeks after which plates were discarded.

$\mathrm{C}$ - The isolated enterobacteriacea were cultured on Chromagar KPC for $18-24 \mathrm{~h}$ at $37^{\circ} \mathrm{C}$. The agar plates were allowed to warm at room temperature before inoculation as they had been refrigerated earlier, then the isolates were streaked onto the plates. After 18-24 h of incubation the plates were examined for typical colony appearance as shown in Table 1 and Fig. 1.

\section{Special safety precaution}

After interpretation all plates were destroyed by autoclaving at $121^{\circ} \mathrm{C}$ for at least $20 \mathrm{~min}$.

3. Modified Hodge Test: Modified Hodge Test (MHT) detects carbapenemase production in isolates of 
Table 1 Typical colony appearance of different isolates on Chromagar-KPC

\begin{tabular}{ll}
\hline Microorganism & Typical colony appearance \\
\hline E.coli & Dark pink to redish \\
Klebsiell, Enterobacter, Citrobacter & Metallic blue \\
Pseudomonas & Cream, translucent \\
Carbapenem sensitive strains & Inhibited \\
Gram + ve strains & Inhibited \\
Yeasts & Mostly inhibited \\
\hline
\end{tabular}

Enterobacteriaceae (According to CLSI guidelines 2012). Carbapenemase production was detected by the MHT(if the test isolate produces the enzyme it allows growth of a carbapenem susceptible strain ( $E$. coli ATCC 25922) towards a carbapenem disk. The result is a characteristic clover leaf-like indentation).

\section{Procedure}

1. A 0.5 McFarland standard suspension of E. coli ATCC 25922 was prepared and inoculated on muller hinton agar as for routine disk diffusion procedure. The plate was allowed to dry 3 to $10 \mathrm{~min}, 10 \mu \mathrm{g}$ meropenem susceptibility disk was placed in the center of the test area.

2. Using a 10- $\mu$ l loop or swab, three to five colonies of tested organisms were inoculated in a straight line from the edge of the disk to the edge of the plat up to four isolates can be tested on the same plate with one drug. The streak should be at least 20 to $25 \mathrm{~mm}$ in length.

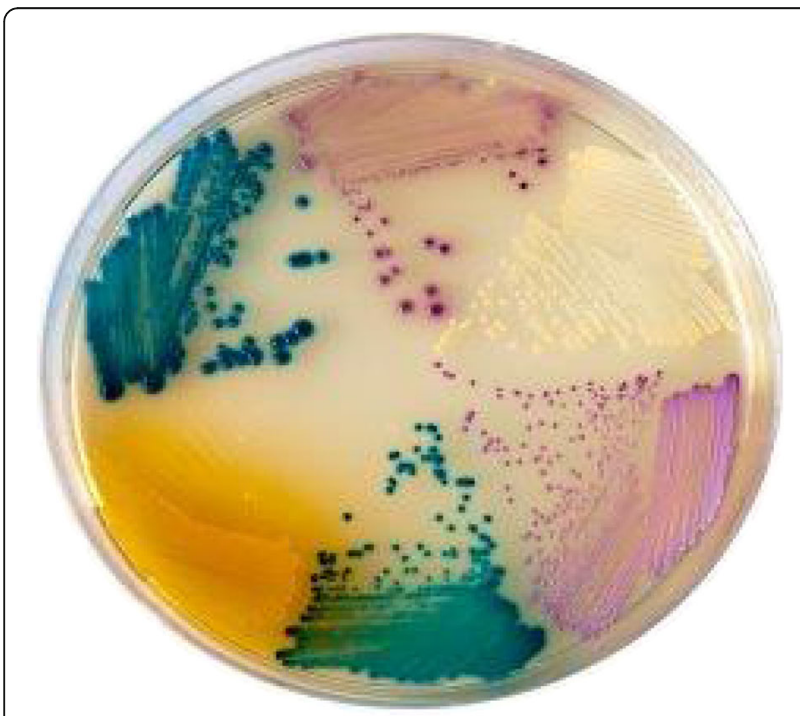

Fig. 1 Typical colony appearance of different isolates on CHROMagar-KPC
3. The plates were incubated overnight at $35 \pm 2{ }^{\circ} \mathrm{C}$ in ambient air for 16-24 h.

After 16-24 h of incubation, the plates were examined for a clover leaf-type indentation at the intersection of the test organism and the E. coli 25922, within the zone of inhibition of the carbapenem susceptibility disk (Clinical and Laboratory Standards Institute. Methods for dilution antimicrobial susceptibility tests for bacteria that grow aerobically and approved standard, 9th ed. CLSI M07-A9. Wayne: Clinical and Laboratory Standards Institute 2012) Fig. 2.

MHT positive test had a clover leaf-like indentation of the E. coli 25922 growing along the test organism growth streak within the disk diffusion zone, indicated that this isolate is producing a carbapenemase.

MHT negative test had no growth of the E. coli 25922 along the test organism growth streak within the disk diffusion zone, indicating that this isolate is not producing a carbapenemase.

4. Genotypic detection of CRE: all clinical isolates of Enterobacteriaceae demonstrating positive Chrom agar or positive Modified Hodge Test were tested for the presence of a blaKPC gene, blaOXA-48 genes, and NDM-1 gene by PCR (Table 2).

\section{1-Extraction of DNA from suspected colony}

Bacterial culture was and the pellet was taken and added protenase $\mathrm{K}$ solution, mixed, lysis solution was added and mixed thoroughly by vortexing to obtain a uniform suspension, sample was incubated at $56{ }^{\circ} \mathrm{C}$ for $10 \mathrm{~min}$ in shaking water bath until the cells were completely lysed then ethanol (96-100\%) was added and mixed, mixture

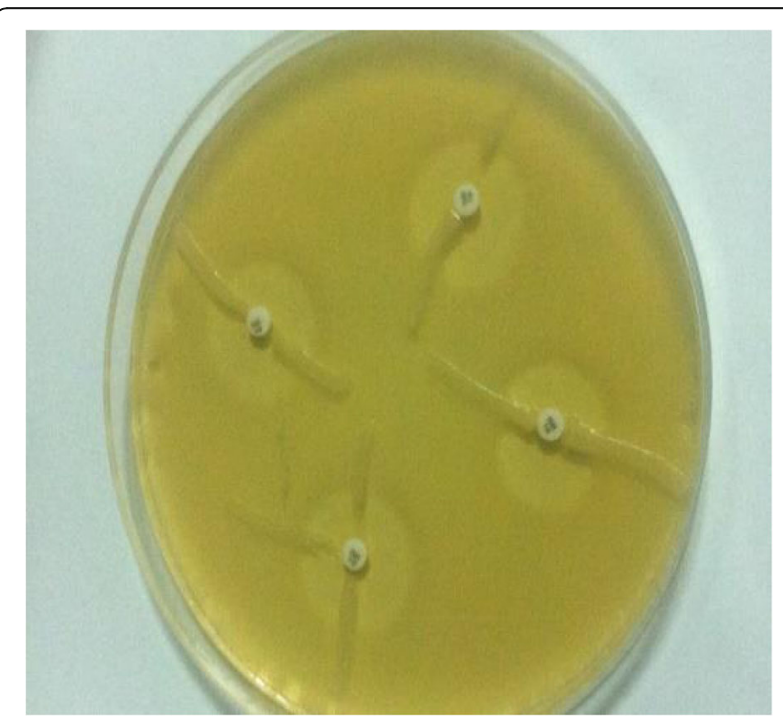

Fig. 2 MHT performed on Muller Hinton agar 
Table 2 Primers for identification of bla KPC gene, bla OXA-48 gene and NDM-1 gene

\begin{tabular}{lll}
\hline & Forward & Reverse \\
\hline BlakPCgene & (ATGTCACTGTATCGCCGTCT) & (TTTTCAGAGCCTTACTGCCC) \\
blaOXA-48 & (TTGGTGGCATCGATTATCGG) & (GAGCACTTCTTTTGTGATGGC) \\
gene & & \\
NDM-1 & (GGTTTGGCGATCTGGTITC) & (CGGAATGGCTCATCACGATC) \\
gene & & \\
\hline
\end{tabular}

was transferred to the spin column and centrifuged then wash using buffer I and buffer II, respectively. Elution buffer was added to the center of the column membrane to elute genomic DNA.

\section{1-Amplification step}

Dream Tag Green PCR Master Mix (Thermo scientific, Germany) was used for PCR. PCR was performed by using the recommended thermal cycling conditions. For blaKPC gene, the PCR conditions were as follows: $15 \mathrm{~min}$ at $95{ }^{\circ} \mathrm{C}$ and 38 cycles of $1 \mathrm{~min}$ at $94{ }^{\circ} \mathrm{C}, 1 \mathrm{~min}$ at $62{ }^{\circ} \mathrm{C}$, and $1 \mathrm{~min}$ at $72{ }^{\circ} \mathrm{C}$. PCR were performed with Dream taq DNA polymerase, and the resulting PCR products were analyzed in $1 \%$ agarose gel with ethedium bromide staining and UV light. For NDM-1 gene, the PCR conditions were as follows: $10 \mathrm{~min}$ at $94{ }^{\circ} \mathrm{C}, 36$ cycles of amplification consisting of $30 \mathrm{~s}$ at $94{ }^{\circ} \mathrm{C}, 40 \mathrm{~s}$ at $52{ }^{\circ} \mathrm{C}$, and $50 \mathrm{~s}$ at $72{ }^{\circ} \mathrm{C}$, and $5 \mathrm{~min}$ at $72{ }^{\circ} \mathrm{C}$ for the final extension. DNA was visualized by electrophoresis in $2 \%$ agarose gel at $100 \mathrm{~V}$ for $1 \mathrm{~h}$ with ethedium bromide staining and UV light. For blaOXA-48 genes, thermal cycler was used under the following conditions: initial denaturation at $49{ }^{\circ} \mathrm{C}$ for $5 \mathrm{~min}$; denaturation at $49{ }^{\circ} \mathrm{C}$ for $60 \mathrm{~s}$; annealing at $56{ }^{\circ} \mathrm{C}$ for $45 \mathrm{~s}$, and extension at $72{ }^{\circ} \mathrm{C}$ for $60 \mathrm{~s}$, repeated for 35 cycles; final extension at $72{ }^{\circ} \mathrm{C}$ for $7 \mathrm{~min}$.

\section{Statistical methodology}

Data were statistically described in terms of mean \pm standard deviation $( \pm \mathrm{SD}$ ), and range, or frequencies (number of cases) and percentages when appropriate. Comparison between the study groups was done using Chi square ( $\left.\chi^{2}\right)$ test was performed. Accuracy was represented using the terms sensitivity, specificity, positive predictive value, negative predictive value, and overall accuracy. $P$ values less than 0.05 was considered statistically significant. All statistical calculations were done using computer programs SPSS (Statistical Package for the Social Science; SPSS Inc., Chicago, IL, USA) version 17 for Microsoft Windows.

\section{Results}

This study was conducted on patients seen at Kasr Alaini hospital and investigated at microbiology laboratory in
Cairo University Hospitals, from May 2013 to October 2013.

The study was conducted on 600 patients; 450 were non hospitalized patients (group I) and came for regular checkup, 150 were inpatients as control group (group II) from different hospital departments. Selection of the patients based on specifically structured questionnaire that addressed certain areas of interest regarding the age, chronic medical illness, previous history of hospitalization within the last two months before study started and if there was any operative history together with antibiotic usage. Patients with any GIT symptoms especially diarrhea were excluded from the study. Based on questionnaire feedback, history and clinical findings, certain patients were suspected to have CPE. Group I includes 337 (56.2\%) males and 113(18.8\%) females and group II includes $83(13.8 \%)$ males and 67(11.2\%) females. Age of group I ranged from 18 to 82 years with a mean of 44 years, while the age of group II ranged from 10 to 77 years with a mean of 37 years. Out of 450 outpatients (group I) fecal samples, 383(85\%) were single isolates included E.coli, klebsiella pneumoniae, Enterobacter and Pseudomonas, while 67(15\%) were combined isolates. Out of the 150 (group II) inpatients fecal samples, $120(80 \%)$ were single isolates included E.coli, klebsiella pneumoniae, Enterobacter, and Pseudomonas, while $30(20 \%)$ were combined isolates. Out of 600 fecal samples (450 from group I and 150 from group II), 12 were positive by disk methods, Chrom agar-KPC, MHT, and PCR(carriage rate $2 \%$ ). Of these twelve positive, two were non-hospitalized patients (group I) (carriage rate $0.33 \%$ ) and ten were hospitalized from different hospital departments (carriage rate 1.7\%).

The positive CPE cases in non hospitalized patients (group I) were two cases $(0.33 \%)$ and they had no history of hospitalization or antibiotic usage within the previous two months, while CPE positive cases in hospitalized patients (10) (group II) had been previously hospitalized in medical wards, and had history of antibiotic usage and seven of them (38.9\%) received treatment with carbapenem antibiotics. Three cases (12\%) in the hospitalized patients (group II) had renal disease (Table 3). All positive CPE cases did not have diabetes millets or operative history. Previous hospitalization and antibiotic

Table 3 Percentage and distribution of CPE cases among group | and group ||

\begin{tabular}{lllll}
\hline Group & Word & $\begin{array}{l}\text { Number } \\
(12)\end{array}$ & $\begin{array}{l}\% \text { from } \\
\text { positive }\end{array}$ & $\begin{array}{l}\% \text { from } \\
\text { total }\end{array}$ \\
\hline Group I(450) & Outpatients & 2 & $16.7 \%$ & $0.33 \%$ \\
Group II(150) & $\begin{array}{l}\text { Different } \\
\text { department }\end{array}$ & 10 & $83.3 \%$ & $1.7 \%$ \\
& $\begin{array}{l}\text { Total } \\
\text { Tot }\end{array}$ & 12 & $100 \%$ & $2 \%$ \\
\hline
\end{tabular}


usage are highly significant with the presence of CPE in group II with $P$ value $<0.001$ (Table 4 ).

All the 12 positive isolates by Chrom agar-KPC and or MHT and PCR had reduced susceptibility to imipenem by disk diffusion while 11 (91.7\%) of them had reduced susceptibility to meropenem by disk diffusion method (Table 5). Chrom-KPC had 100\% sensitivity and 100\% specificity as Modified Hodge Test (MHT). All positive isolates by Chrom-KPC tested for carbapenemase production by the MHT, 8 (61.5\%) isolates were positive by (MHT) 2 from group I and 6 from group II, while 5 $(38.5 \%)$ isolates were negative by (MHT) 1 from group I and 4 from group II (Fig. 3). MHT had sensitivity of $66.7 \%$ and specificity of $100 \%$. Chrom-KPC had $100 \%$ sensitivity and $100 \%$ specificity while MHT had sensitivity $66.7 \%$ and specificity $100 \%$.

\section{Genotypic detection of KPC producing isolates}

All fecal samples proved positive by Chrom agar KPC were tested by convential PCR. According to PCR results, 9 (75\%) isolates were positive for NDM (2 from group I and 7 from group II) and 5 (41.7\%) isolates were OXA positive (all of them are from group II) while all the positive isolates (12) were KPC negative as shown in Table 6 and Fig. 4.

\section{Discussion}

CPE are gram-negative bacteria that are resistant to carbapenem class of antibiotics, and they are resistant because they produce an enzyme called a carbapenemase that disables the drug molecule. The resistance can vary from moderate to severe. Enterobacteriaceae are common commensals and infectious agents. The bacteria can kill up to half of patients who get bloodstream infections (Tom Frieden, head of the Centers for Disease Control has referred to CPE as "nightmare". Spread of CPE (carbapenemase producing Enterobacteriaceae) has been increasingly reported worldwide (Queenan and Bush 2007a). Treatment options for infection due to these multi-drug resistant organisms are extremely limited and effective therapy may be delayed while microbiology laboratory confirmatory results are awaited (Burns and Schaffer 2011).

Outbreaks of CPE, primarily Klebsiella pneumoniae, have been reported in the USA since the beginning of the 2000s and recently in several other regions worldwide. These isolates are resistant to all carbapenems and susceptible only to colistin and gentamicin. At the same time, sporadic isolates of Enterobacteriaceae that were resistant to meropenem (MEM) but sensitive to imipenem (IPM) emerged (Nordman et al. 2012). Given the limited therapeutic options available, the accurate and timely detection of KPC-producing Enterobacteriaceae is vital in order to control their spread (Nordmann et al. 2009). The aim of our study is to evaluate the incidence of intestinal colonization with CPE in non-hospitalized patients and to assess a microbiological protocol for screening these isolates in fecal material. In this study, 600 fecal samples were collected, 450 of them from out patients clinics (group I) and 150 from different hospital departments (group II). Among these isolates, 12 patients $(2 \%)$ had reduced susceptibility to one or more carbapenems and positive Chromagar KPC and or positive Modified Hodge Test, $2(0.3 \%)$ of them were

Table 4 Significance of risk factors in inpatients (group II)(150) to CPE

\begin{tabular}{|c|c|c|c|c|c|c|}
\hline & & \multicolumn{4}{|c|}{ Culture.chrom-KPC } & \multirow[t]{3}{*}{$P$ value } \\
\hline & & \multicolumn{2}{|l|}{-ve } & \multicolumn{2}{|l|}{ +ve } & \\
\hline & & Count & $\%$ & Count & $\%$ & \\
\hline \multirow[t]{2}{*}{$\mathrm{DM}$} & No & 132 & $92.9 \%$ & 10 & $7.1 \%$ & \\
\hline & Yes & 8 & $100.0 \%$ & & & 1.0 \\
\hline Group total & & 140 & $93.3 \%$ & 10 & $6.7 \%$ & Non significance \\
\hline \multirow[t]{2}{*}{ Renal } & No & 118 & $94.4 \%$ & 7 & $5.6 \%$ & \\
\hline & Yes & 22 & $88.0 \%$ & 3 & $12.0 \%$ & 0.37 \\
\hline Group total & & 140 & $93.3 \%$ & 10 & $6.7 \%$ & Non significance \\
\hline \multirow[t]{2}{*}{ Previous hospitalization } & No & 140 & $95.9 \%$ & 6 & $4.1 \%$ & \\
\hline & Yes & & & 4 & $100.0 \%$ & $<0.001^{* *}$ \\
\hline Group total & & 140 & $93.3 \%$ & 10 & $6.7 \%$ & Highly significant \\
\hline \multirow[t]{3}{*}{ Antibiotic groups } & No & 22 & $100.0 \%$ & & & \\
\hline & Others & 107 & $97.5 \%$ & 3 & $2.5 \%$ & $<0.001^{* *}$ \\
\hline & Tinam and meronem & 11 & $61.1 \%$ & 7 & $38.9 \%$ & Highly significant \\
\hline Group total & & 140 & $93.7 \%$ & 10 & $6.3 \%$ & $100.0 \%$ \\
\hline
\end{tabular}

${ }^{*} P$ value is significant $<0.05,{ }^{*}$ highly significant 
Table 5 Comparison between sensitivity and specificity of Chrom-KPC and MHT

\begin{tabular}{lllll}
\hline & Sensitivity & Specificity & PPV & NPV \\
\hline Chrom-KPC & $100 \%$ & $100 \%$ & $100 \%$ & $100 \%$ \\
MHT & $66.7 \%$ & $100 \%$ & $100 \%$ & $20 \%$ \\
\hline
\end{tabular}

Sensitivity $(\mathrm{MHT}) 8 / 12=66.7 \%$, specificity $=1 / 1=100 \%$,

PPV $=8 / 8=100 \%$, NPV $=1 / 5=20 \%$

non hospitalized (group I|) while 10 (1.7\%) were hospitalized (group II). The frequency of CPE fecal colonization in our study is higher than the clinical prevalence in Spanish hospitals (1.1\%) although it is still lower than that reported in another study as in hospitalized patients in France during a non-outbreak period CPE was (5.3\%) (Miro and et al. 2010) Out of the 600 fecal samples, 12 were positive by both Chrome culture and PCR. 11 of them were typical $K$. pneumoniae showing mucoid metallic blue colonies on Chromagar KPC and one isolate was E.coli with dark pink colonies.

Chromagar KPC has sensitivity of $100 \%$, specificity of $100 \%$, PPV of $100 \%$, NPV of $100 \%$ all in relation to PCR as gold standard. This results in agreement with the study done by Samra et al. that represented sensitivity and specificity for Chromagar KPC in relation to PCR as 100 and 98.4\%, respectively (Samra et al. 2008). The 12 fecal samples had reduced susceptibility to one or more carbapenems according to CLSI guidelines 2012 using disk diffusion, $12(100 \%)$ of isolates had reduced susceptibility to imipenem while $11(91.7 \%)$ had reduced susceptibility to meropenem. This can be explained by Thomson et al., who stated that low-level resistance and even susceptibility to carbapenems have been observed for producers of any type of carbapenemase (Thomson 2010) while Kanj, Souha et al. stated that various studies have reported that carbapenem-resistant bacteria may be incorrectly identified as carbapenem susceptible, resulting in inappropriate selection of therapy (Kanj and Kanafani 2011). The 12 fecal samples had reduced susceptibility to one or more carbapenems and positive Chromagar- KPC or positive Modified Hodge Test, 2 $(0.3 \%)$ of them were from outpatients clinics while, 10 (1.7\%) were inpatients and they were mainly from ICU (50\%) and neurology departments (20\%) while in the study done by Desiree et al. their positive isolates mainly from neurology and nephrology departments. (Desiree et al. 2012) In our study,11 (91.7\%) among positive cases were Klebsiella pneumoniae and 1 (8.3\%) was E. coli. On the other hand a study done by Desiree et al. showed that the most common Enterobacteriaceae identified from fecal samples were (72.7\%) Klebsiella pneumoniae (18.2\%) were Enterobacter cloacae and (9.1\%) were E. coli. (Desiree et al. 2012). All the positive isolates from non-hospitalized patients had no history of previous hospitalization or antibiotic usage while, hospitalized patients had history of antibiotic usage and seven of them (38.9\%) received treatment with carbapenem antibiotics. On the other hand, a study done by Desiree et al. showed that only one from non hospitalized patients had previous history of hospitalization (Desiree et al. 2012). The rapid detection of KPC-producing Enterobacteriaceae is of great importance as some studies have indicated. In our study, Carbapenemase production was also detected by the MHT. Eight isolates (two from group I and six from group II) displayed positive results in the MHT and convential PCR (true positive) while, four isolates displayed negative MHT and positive PCR (false negative) and 1 isolates displayed negative MHT and negative PCR (true negative) MHT has sensitivity of $66.7 \%$, specificity of $100 \%$, PPV of $100 \%$, NPP of $20 \%$ all in relation to PCR as gold standard (Kalpoe et al. 2011)

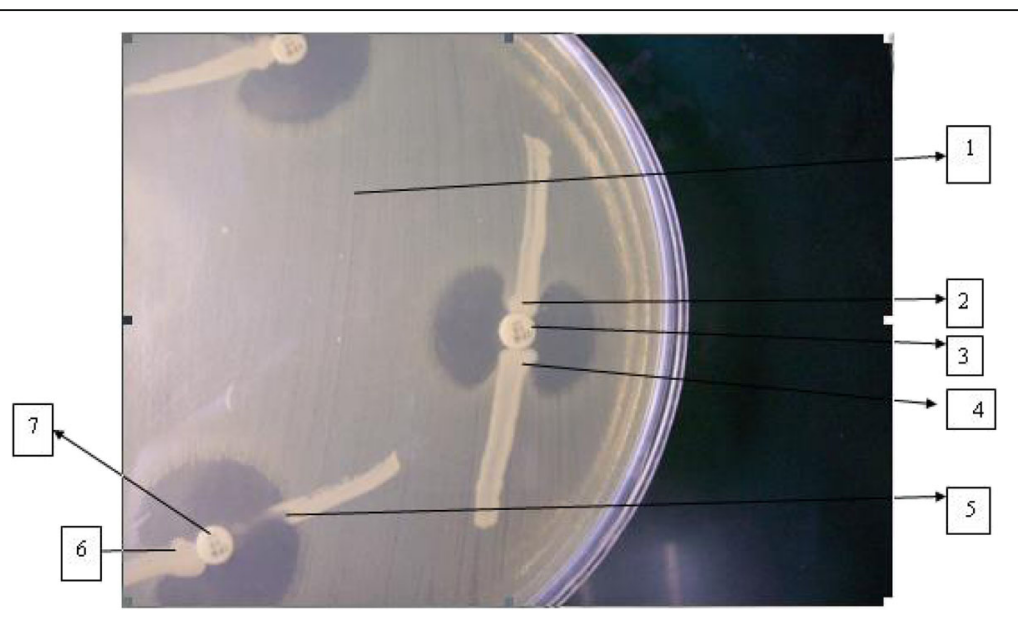

Fig. 3 MHT Modified Hodge Test performed on Muller Hinton agar plate. 1 is E coli ATCC 25922, 2, 4, and 6 are positive cases; 3, 7 are carbapenem disks, and 5 is a negative case 
Table 6 Results of PCR test among positive cases

\begin{tabular}{lllllll}
\hline & KPC & $\%$ & NDM & $\%$ & OXA & $\%$ \\
\hline $\begin{array}{l}\text { Outpatients group I } \\
(n=2)\end{array}$ & 0 & $0 \%$ & 2 & $16.7 \%$ & 0 & $0 \%$ \\
$\begin{array}{l}\text { Inpatients group II } \\
(n=10)\end{array}$ & 0 & $0 \%$ & 7 & $58.3 \%$ & 5 & $41.7 \%$ \\
Total (12) & 0 & $0 \%$ & 9 & $75 \%$ & 5 & $41.7 \%$ \\
\hline
\end{tabular}

reported that the Modified Hodge Test based on in vivo production of carbapenemase has been suggested for detecting carbapenemase producers. Also as reported by (Pasteran et al. 2009), MHT is sometimes difficult to interpret, and false-positive results have been reported (Kalpoe et al. 2011; Pasteran et al. 2009). In another study done at Centers for Disease Control and Prevention in Atlanta GA in 2007, 45 isolates (26 of K. pneumoniae, 9 of $K$. oxytoca, and 10 of E. coli) were evaluated by Modified Hodge test and all of them were validated by PCR for the detection of KPC activity with $100 \%$ sensitivity and specificity (Anderson et al. 2007). This shows that Modified Hodge test is a very sensitive and reliable test for detection of carbapenemases. A study was carried out in Greece in 2007 to evaluate different laboratory tests for detection of MBLs in Enterobacteriacae. Modified Hodge test detected 98\% cases keeping PCR as the gold standard while only $0.03 \%$ was detected as false positive (Galani et al. 2008). In our study, two of the positive isolates detected in non hospitalized patients (group I) by conventional PCR were NDM producers (16.7\%) and theses positive isolates include E.coli and $K$. pneumoniae while in hospitalized patients (group II) 10 positive isolates were NDM producers $(58.3 \%)$ and $(41.7 \%)$ were OXA-48 producers but not KPC producers and these isolates were $K$. pneumoniae, while on the study done by Desiree et al. they were detect NDM and KPC producers but not OXA-48 producers (Desiree et al. 2012). In conclusion,

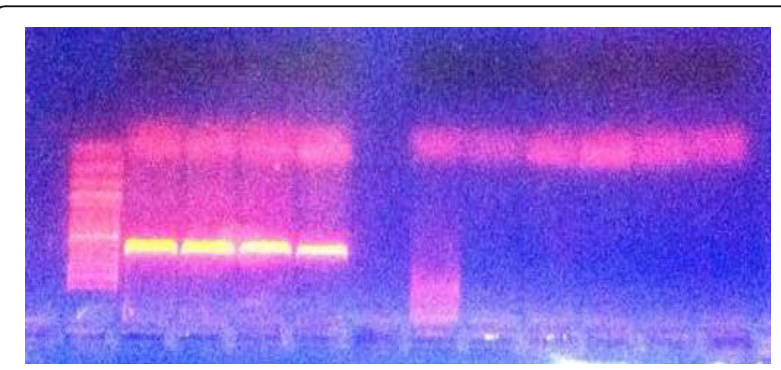

Lane 1: PCR marker ladder (50-500 bp),

Lanes 2, 3, 4 and 5: NDM positive

Lanes 6, 7, 8, 9, 10, 11: KPC negative

Fig. 4 Restriction enzyme analysis and agarose gel electrophoresis of CPE we describe a fecal carriage of CPE; all of them are NDM producers or OXA-48 producers in patients non-infected by these organisms in Kasr Alaini hospital. These results are an alert for a hidden fecal carriage of patients with $\mathrm{CPE}$ that would not have been detected as most of them were not infected by these organisms. Standard for identification of carbapenemases is based on use of molecular techniques; mostly PCR which when performed on colonies may give results within 4-6 h with excellent sensibility and specificity. The main disadvantages of molecular-based technologies for detection of carbapenemases are their cost, the requirement of trained personnel, and the absence of detection of any novel carbapenemase gene. Thus, there is an urgent need for an inexpensive, rapid, sensitive, and specific test for detection of carbapenemase activity (Nordmann et al. 2011).

\section{Conclusions}

The results are an alret for a hidden fecal carriage of patients with CPE that would not have been detected as most of them were not infected by the organisms.

\section{Recommendations}

Spread of carbapenemase-producing enterobacteriaceae (CPE) has been increasingly reported worldwide. We recommended that every microbiology laboratory should be able to undertake their screening, using carbapenem disk diffusion or Chromagar-KPC, which showed agreement with each other for detection of carbapenem susceptibility. We recommend sequencing for accurate detection of all carbapenemase encoding genes (both KPC and VIM type).

Every microbiology laboratory should be resourced with adequate staff to conduct and maintain CPE screening and most importantly in the event of an outbreak as this type of resistance is usually associated with bad outcome and high mortality rate. Appropriate antimicrobial prescribing and good infection prevention and control practices are essential to prevent the emergence of CPE in all healthcare settings. The association of previous antibiotic intake especially carbapenems among cases infected with CPE necessitates that hospitals should ensure that they have active antimicrobial stewardship programs, this should include restriction of carbapenem use. Carbapenems should only be prescribed following consultation with a clinical microbiologist or infectious diseases physician.

\section{Acknowledgements}

We thank all the lab staff that worked with us to complete our study.

\section{Authors' contributions}

All authors had substantial contributions to the conception and design, acquisition of data, analysis and interpretation of the data. AS drafted the article and revised it critically for important intellectual content. All authors have approved of the final version to be published, and agreement was obtained to be accountable for all aspects of the work in ensuring that questions related to the accuracy or integrity of any part of the work are appropriately investigated and resolved. 


\section{Competing interests}

None.

\section{Publisher's Note}

Springer Nature remains neutral with regard to jurisdictional claims in published maps and institutional affiliations.

Received: 10 January 2017 Accepted: 7 March 2017

Published online: 18 March 2017

\section{References}

Anderson KF, Lonsway RD, Rasheed KJ, Biddle J, Jensen B, McDougal LK, et al. Evaluation of methods to identify the Klebsiella pneumoniae carbapenemase in Enterobacteriaceae. J Clin Microbiol. 2007:45:2723-5.

Burns K. and Schaffer K. CRE update issued on laboratory detection and infection control measures. Epi Insight. 2011;12(5).

CDC. CRE toolkit: facility guidance for control of carbapenam-resistent Enterobactericae (CRE). Atlanta; CDC; Department of health and human services; 2015.

Clinical and Laboratory Standards Institute. Methods for dilution antimicrobial susceptibility tests for bacteria that grow aerobically: approved standard, 9th ed. CLSI M07-A9. Wayne: Clinical and Laboratory Standards Institute; 2012.

Cornaglia G, Giamarellou H, Rossolini GM. Metallo-beta-lactamases: a last frontier for beta-lactams? Lancet Infect Dis. 2011;11:381-93.

Desiree $G$, et al. Fecal carriage of carbapenemase-producing Enterobacteriaceae: a hidden reservoir in hospitalized and non hospitalized patients. J Clin Microbiol. 2012;10:1128.

Galani I, Rekatsina DP, Hatzaki D, Plachouras D, Souli M, Giamarellou H. Evaluation of different laboratory tests for the detection of metallo- $\beta$-lactamase production in Enterobacteriaceae. J Antimicrob Chemother. 2008;61:548-53.

Grundmann $\mathrm{H}$, et al. Carbapenem-non-susceptible Enterobacteriaceae in Europe: conclusions from a meeting of national experts. Euro Surveill. 2010;15(46).

Kalpoe JS, Al Naiemi N, Poirel L, Nordmann P. Detection of an Ambler class D OXA-48-type $\beta$-lactamase in a Klebsiella pneumoniae strain in The Netherlands. J Med Microbiol. 2011:60:677-8.

Kanj S, Kanafani ZA. Current concepts in antimicrobial therapy against resistant gram-negative organisms: extended-spectrum $\beta$-lactamase-producing Enterobacteriaceae, carbapenem-resistant enterobacteriaceae, and multidrugresistant pseudomonas aeruginosa. Mayo Clin Proc. 2011;86(3):250-9. doi:10.4065/mcp.0674.

Miro E, et al. Estudio de la prevalencia de $\beta$-lactamases AmpC plasmidicas y carbapenemasas en enterobacterias en Espana. XIV Congreso SEIMC. 2010. p. 706.

Nordmann P, Dortet L, Poirel L. Carbapenem resistance in Enterobacteriaceae: here is the storm. Trends Mol Med. 2012;18(5):263-72. doi:10.1016/j.molmed. 2012.03.003.

Nordmann P, Cuzon G, Naas T. The real threat of Klebsiella pneumoniae carbapenemase producing bacteria. Lancet Infect Dis. 2009;9:228-36.

Nordmann P, Poirel L, Carrër A, Toleman MA, Walsh TR. How to detect NDM-1 producers. J Clin Microbiol. 2011;49:718-21.

Pasteran F, Mendez T, Guerriero L, Rapoport M, Corso A. Sensitive screening tests for suspected class A carbapenemase production in species of Enterobacteriaceae. J Clin Microbiol. 2009;47(6):1631-9.

Queenan AM, Bush K. Carbapenemases: the Versatile B-Lactamase. Clin Microbiol Rev. 2007a;20:440-58.

Ruppé E, et al. Development of a phenotypic method for detection of fecal carriage of OXA-48-producing Enterobacteriaceae after incidental detection from clinical specimen. J Clin Microbiol. 2011:49:2761-2762.12.

Samra Z, Bahar J, Madar-Shapiro L, Aziz N, Israel S, Bishar J. CHROMagar for carbapenem resistant Enterobacteriaceae. J Clin Microbiol. 2008:46:3110-1.

Sánchez-Romero I, et al. Nosocomial outbreak of VIM-1-producing Klebsiella pneumoniae isolates of multilocus sequence type 15: Molecular basis, clinical risk factors, and outcome. Antimicrob Agents Chemother. 2012;56:420-7.

Solé $\mathrm{M}$, et al. First description of an Escherichia coli strain producing NDM-1 carbapenemase in Spain. Antimicrob Agents Chemother. 2011;55:4402-4.

Tato $\mathrm{M}$, et al. Carbapenem hetroresistance in VIM-1-producing Klebsiella pneumoniae isolates belonging to the same clone: consequences for routine susceptibility testing. J Clinmicrobiol. 2010;48:4089-93.

Thomson KS. Extended-spectrum $\beta$-lactamase, AmpC and carbapenemase issues. J Clin Microbiol. 2010;48:1019-25.
Tórtola MT, et al. First detection of acarbapenem-hydrolyzing metalloenzyme in two Enterobacteriaceae isolates in Spain. Antimicrob Agents Chemother. 2005:49:3492-4.

Yong MA, Toleman CG, Giske HS, Cho K, Sundman K, Lee TR. Walsh characterization of a new metallo-beta-lactamase gene, bla(NDM-1), and a novel erythromycin esterase gene carried on a unique genetic structure in Klebsiella pneumoniae sequence type 14 from India. Antimicrob Agents Chemother. 2009:53:5046-505.

\section{Submit your manuscript to a SpringerOpen ${ }^{\circ}$ journal and benefit from:}

- Convenient online submission

Rigorous peer review

- Immediate publication on acceptance

- Open access: articles freely available online

- High visibility within the field

- Retaining the copyright to your article

Submit your next manuscript at $\boldsymbol{s p r i n g e r o p e n . c o m ~}$ 